\title{
MINERALIZAÇÃO E SORÇÃO DE ATRAZINA EM LATOSSOLO ROXO SOB CULTIVO CONVENCIONAL E PLANTIO DIRETO(1)
}

\author{
M. A. ALBUQUERQUE(2), C. E. G. R. SCHAEFE ${ }^{(3)}$, J . M. FOLONI $^{(4)}$, \\ J.C. KER ${ }^{(3)} \&$ L. E. F. FONTES $S^{(3)}$
}

\begin{abstract}
RESUMO
A atrazina, herbicida do grupo das s-triazi nas, foi utilizada em experimentos de laboratório para estudos de mineralização e sor ção em amostras de Latossolo R oxo sob cultivo convencional e plantio direto. Atrazina- ${ }^{14} \mathrm{C}$ marcada no anel de triazina foi adicionada às amostras de solos, sendo $0{ }^{14} \mathrm{CO}_{2}$ produzido e os extratos de atrazina- ${ }^{14} \mathrm{C}$ do solo anal isados por cintilação líquida. Os resultados mostraram taxas de mineralização de atrazina muito baixas, apresentando correlações si gnificativas com os teores de carbono, nitrogênio total e frações húmicas do solo. A mineralização de atrazina decaiu em profundidade, e o Latossolo Roxo sob sistema plantio direto mostrou maior mineralização de atrazina em virtude dos maiores teores de car bono. E m contrapartida, a fase lag do plantio convencional foi mais curta, evidenciando maior adaptação microbiana nos solos sob manejo convencional, onde o her bicida é mais intensamente utilizado em pré-emergência. Com a adição de substratos orgânicos, houve aumento no processo de mineralização de atrazi na. Verificou-se que a atrazi na contém um componente rápido de sorção no Latossolo Roxo, em torno de duas horas, e um componente mais lento que se estabelece até $12 \mathbf{~ h}$. A matéria orgânica do solo foi o principal fator responsável pela sorção da atrazi na ( $50 \%$ da atrazina adicionada) ao longo do tempo, enquanto os óxidos de ferro/alumínio e minerais de argila 1:1 não contribuíram, de forma significativa, para a sorção ( $5 \%$ da atrazina adicionada). Solos com baixo teor de matéria orgânica podem não reter atrazina em quantidades suficientes para evitar a contami nação subsuperficial, ondea molécula não pode ser mineralizada, trazendo riscos de contami nação do lençol freático.
\end{abstract}

Termos de indexação: biodegradação, adsorção, oxissolos, her bicida.

(1) Recebido para publicação em fevereiro de 2000 e aprovado em setembro de 2000.

(2) Professora da Fundação Educacional de Caratinga - FUNEC. Av. Moacyr de Mattos 49, CEP $35300-047$ Caratinga (MG). Bolsista CNPq.

(3) Professor do Departamento de Solos, Universidade Federal de Viçosa - UFV. CEP 36571-000 Viçosa (MG). Bolsista CNPq.

(4) Engenheiro-Agrônomo, Departamento de Solos, UFV. 


\title{
SUMMARY: MINERALIZATION AND SORPTION OF ATRAZINE IN A DUSKY-RED LATOSOL UNDER CONVENTIONAL AND NO- TILLAGE SYSTEMS
}

\begin{abstract}
Atrazine, an herbicide of the s-triazine group, was used in laboratory experiments to determine its mineralization and sorption on samples of a Dusky-Red Latosol under conventional and no-tillagesystems. Atrazinering-label ed ${ }^{14} \mathrm{C}$ was added to thesoil samples, with the ${ }^{14} \mathrm{CO}_{2}$ being produced and atrazine ${ }^{14} \mathrm{C}$ soil extracts analyzed by liquid scintillation. Theresults showed very low mineralization rates of atrazine, which werecorrelated with the total carbon and nitrogen contents, as well as with soil humicfractions. Atrazinemineral ization decreased with depth, whereas the soil under no-tillage system showed higher atrazine mineralization compared with the conventional system, due to higher amounts of organic matter in theformer. On theother hand, thelag phase of the conventional system was shorter, indicating a greater adaptation of themicrobial community to degradeatrazinein soils where application of this herbicide is a common preemergence practice. The addition of organic substrates resulted in increasing atrazi nemi neralization. It was al so verified that atrazine had a short-term sorption component of two hours, foll owed by a slower component established up to 12 hours. Thesoil organic matter was themain factor control ling atrazinesorption (50\% of added atrazi ne), whereas iron/ al umi num oxi des and 1:1 d ay mineral s had no si gnificant contribution to thesorption process (about $5 \%$ of theadded atrazine). Soils with low organic matter content are not able to absorb enough atrazi ne to avoid subsurface contami nation, resulting in serious risks of watertablepollution.
\end{abstract}

Indexterms: biodegradation, adsorption, Oxisols, herbicide.

\section{INTRODUÇÃO}

A atrazina (2-cloro-4-etilamino-6-isopropilamino-striazina) éum herbicida pertencente ao grupo das striazinas. É um póincol or com ponto defusão de $176^{\circ} \mathrm{C}$ com solubilidade em água de $33 \mathrm{mg} \mathrm{L}^{-1}$, sendo considerado como uma base fraca com pKa $=1,7$ (Worthing \& Hance, 1991). Sua utilizaçãointensiva ebaixa degradabilidadetêm levado ao acúmulo desse composto no ambiente, contaminando águas de superfície e lençol freático (Ritter et al., 1994). A permanência deste composto no sol o é variável, com valores de meia-vida entre 37 dias até 3-5 anos (Armstrong et al., 1967). Essa variabilidade deve-se às diversas condições eclasses de solos estudados.

O processo de mineralização e a adsorção são os principais fatores que controlam o destino final da atrazina no solo. O estudo de sorção é de grande importância para a compreensão da retenção deste composto pel os col ói des do sol o, os quais influenciam o processo de lixiviação, volatilização emineral ização, sendo também fundamental nas definições de doses a serem utilizadas (Bailey \& Whitte, 1970).

A degradação microbiana pode ser bastante expressiva para a inativação da atrazina como herbicida, mas seus metaból itos podem persistir por longo tempo no solo (Bollag \& Liu, 1990; Aislabie \& Lloyd-J ones, 1995). Quandoa degradaçãoécompleta, levando à formação de $\mathrm{CO}_{2}, \mathrm{NH}_{3} \mathrm{eH}_{2} \mathrm{O}$, o processo é chamado de mineral ização.
A natureza dos colóides do solo determina as interações das moléculas dos pesticidas e as frações orgâni cas e minerais do solo. Para o estabel ecimento destas ligações em compostos não-iônicos (como a atrazina), segundo Green \& Karikhoff (1990), são necessárias horas, até mesmo dias, e a matéria orgânica constitui o principal componenteno processo deadsorção.

Para a maioria das aplicações práticas de adsorção em laboratório, convencionou-se o tempo de $24 \mathrm{~h}$, tempo este considerado por muitos autores suficiente para obtenção do coeficiente de adsorção. Green \& Karikhoff (1990) relatam que este equilíbrio pode não ser al cançado, quando se leva em consi deração a adsorção nos locais internos dos colóides.

O tempo que um pesticida, sobretudo herbicida, leva para ser adsorvido pelos colóides do solo é importante para o manejo, devido ao efeito de fitotoxidez, lixiviaçãoeescorrimento superficial desses produtos pela chuvas.

Os objetivos deste experimento foram: avaliar a mineralização da atrazina em Latossolo Roxo sob manejo convencional e plantio direto em diferentes profundidades, avaliar a cinética de adsorção de atrazina e verificar o tempo necessário para estabel ecimento de equilíbrio na obtenção dos coeficientes desor ção para Latossol o Roxo, sob manejo convencional e plantio direto, com e sem matéria orgânica. 


\section{MATE RIAL E MÉTODOS}

\section{Localização e coleta do solo}

Amostras de um Latossol o Roxo foram coletadas no município de Guaíra (SP), em duas condições de cultivo intensivo sob pivô central, uma com plantio convencional há mais de 30 anos (F azenda Coqueiro) e outra com plantio direto há mais de 14 anos (F azenda Lagoa-do-F ogão).

Para os experimentos demineral izaçãoe adsorção, as amostras de solo foram coletadas em três profundidades $(0-10,30-40$ e $70-80 \mathrm{~cm})$, utilizando tubos de PVC introduzidos no perfil do solo. Após a coleta, as amostras foram armazenadas em refrigerador a 4C para posterior utilização nos experimentos.

\section{Caracterização química, física, mineralógica e micromorfológica dos solos}

A análise textural foi realizada pelo método da pipeta, conformeE MBRAPA (1997). Para identificar os minerais presentes, a argila total foi analisada por difratometria de raios- $X$, em difratômetro com tubo de cobre efiltro de níquel, na faixa de 4a 40 을.

As amostras de solo, terra fina seca ao ar (TFSA), foram submetidas às análises químicas de rotina. A densidade aparente foi obtida por meio do método do torrão parafinado. Todos os métodos da análise de rotina e densidade aparente encontram-se descritos em EMBRAPA (1997). O fracionamento das substâncias húmi cas seguiu a técnica da solubilidade diferencial, utilizando os conceitos defrações húmicas, estabelecidas pela "I nternational Humic Substances Society', descritas por Hayes et al. (1989), e quantificadas juntamente com o carbono total pelo método de Yeomans \& Bremner (1988). O nitrogênio foi quantificado pelo método Kjeldahl, conforme EMBRAPA (1997). Todas as análises decaracterização foram realizadas em triplicatas.

As seções finas dos sol os estudados foram obti das a partir de amostras indeformadas de $10 \times 5 \mathrm{~cm}$ e impregnadas. A descrição das lâminas seguiu as recomendações deF itzpatrick (1993), para estudos de microestrutura e porosi dade.

\section{Herbicida utilizado}

Nos estudos de mineralização, foi utilizada a atrazina comercial-Ciba Agro, enquanto, nos experimentos de sorção, foi utilizada uma atrazina grau-analítico (98\%). E m diluição, juntamente com a atrazina comercial ou pura, foi utilizada a Atrazina14C marcada no anel de triazina (Ciba Agro, 3,5 MBq mmol-1, 94\% de pureza radioquímica). A atrazina- ${ }^{14} \mathrm{C}$ foi utilizada para aumentar a precisãoe segurança nas anál ises da mineral ização a ${ }^{14} \mathrm{CO}_{2}$, na determinação dos remanescentes das soluções de equilíbrio. A radiotividade foi analisada por meio da técnica decintilaçãolíquida descrita por Albuquerque (1995).

\section{Mineralização de atrazina no solo}

O aparato respirométrico, adaptado por Albuquerque (1994), foi utilizado nos estudos de mineralização de atrazina. Os tratamentos foram realizados em triplicatas, constituídos de $50 \mathrm{~g}$ de amostras de Latossol o Roxo (manejo convencional e plantio direto), nas profundidades de 0-10, 30-40 e 70-80 cm, acondicionados em frascos E rlenmyers de $125 \mathrm{~mL}$, que continham $10 \mu \mathrm{g}$ de atrazina comercial +atrazina- ${ }^{14} \mathrm{C}$. O sistema foi alimentado por um fluxo de ar originado por uma bomba de aquário. Esse fluxo de ar passou por duas colunas com soda-lime (Sigma) para retirada do $\mathrm{CO}_{2}$, e, a seguir, por um recipiente de $\mathrm{NaOH} 0,1 \mathrm{~mol} \mathrm{~L}^{-1} \mathrm{e}$, em outrofrasco comágua, para manutenção da umidade das amostras de solo próxima da capacidade de

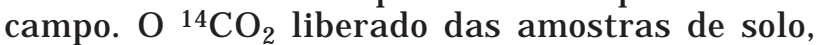
decorrente da mineralização da atrazina- ${ }^{14} \mathrm{C}$, foi capturado em solução de $\mathrm{NaOH}$ 0,1 mol L-1.

Alíquotas de $1 \mathrm{~mL}$ da solução de $\mathrm{NaOH} 0,1 \mathrm{~mol} \mathrm{~L}^{-1}$ (armadilha do ${ }^{14} \mathrm{CO}_{2}$ ) foram coletadas de todos os tratamentos após 9, 16, 30, 41, 62, 100, 106, 119 e 127 dias de incubação do experimento e depositadas em frascos de cintilação líquida, nos quais $7 \mathrm{~mL}$ de coquetel cintilante (Sigma) foram adicionados para análiseda radioatividade em Contador de Cintilação Líquida (Beckman, model o LS6000I C).

Após 100 dias de incubação, diferentes substratos orgânicos foram adicionados nas amostras de solo para avaliar o seu efeito na mineralização de atrazina. Não houve possibilidade de se utilizarem dois tipos de substratos para os dois solos (convencional eplantio direto) em virtude do número de repetições. As amostras de solo sob plantio convencional ( $\mathrm{Faz}$. Coqueiro) receberam 1,5 g de composto orgânico e, sob plantio direto ( $F$ az. Lagoa do Fogão), 1,5 g de amido solúvel.

Os teores de carbono total, juntamente com os teores de nitrogêni o total do solo, foram comparados coma mineral ização deatrazina aos 62 dias, utilizando correlações dePearson. Os resultados demineralização foram submetidos à anál ise de variância, utilizando o Sistema de Anál ise Estatística eGenética (SAEG 5.0) da UniversidadeF ederal deViçosa.

\section{Cinética de adsorção de atrazina no solo}

Foram utilizadas amostras de Latossolo Roxo com os seguintes tratamentos: sol o integral (sol), sol o com matéria orgânica removida (sols/mo), argila com matéria orgânica (argc/mo), argila com matéria orgânica removida (args/mo) e argila com óxido de ferro removido (args/fe). A fração argila foi obtida a partir do fracionamento textural dos solos, sendo separada com base no princípio da lei deStokes. Para facilitar a dispersão da argila, foi utilizada água destilada com pH 10, ajustada com carbonato de sódio. Após o tempo de sedimentação adequado, a suspensão de argila foi sifonada para recipiente próprio. Este procedimento foi repetido várias vezes 
até se obter material suficiente para o experimento. $\mathrm{O} \mathrm{pH}$ da suspensão de argila foi abaixado para 4,0 com $\mathrm{HCl}$, para provocar a floculação. Depois de removido o excesso de sobrenadante, a argila foi seca emestufa a $60^{\circ} \mathrm{C}$, mó da ehomogeneizada em almofariz.

A eliminação da matéria orgânica foi feita com hipoclorito de sódio de acordo com método descrito por Anderson (1963) ea el iminação de óxido de ferro com ditionito-citrato (Mehra \& J ackson, 1960). Nestes tratamentos, as amostras sofreram novo tratamento com hipocl orito de sódio para el iminação de citrato. As amostras que sofreram tratamento foram lavadas com água destilada e centrifugadas várias vezes para eliminação de excesso de sódio. Neste processo delavagem, foram real izadas leituras deste el emento nas sol uções de lavagem, com vistas em controlar sua concentração nas amostras. O pH de todas as amostras foi ajustado para 6,0, utilizando $\mathrm{NaOH}$ 0,01 eHCl 0,01 mol L-1. A pós esteprocedimento, as amostras foram secas em estufa a $60^{\circ} \mathrm{C}$, trituradas em al mofariz ecolocadas em dessecador.

Parcelas com 2,5 $\mathrm{cm}^{3}$ dos materiais de solo foram acondicionadas em frascos Erlenmyers de $50 \mathrm{~mL}$, nos quais foram adicionados $7,5 \mathrm{~mL}$ de solução $\mathrm{CaCl}_{2}$ $0,001 \mathrm{~mol} \mathrm{~L}^{-1}$ que continha $5 \mathrm{mg}$ de atrazina (grau analítico + atrazina- ${ }^{14} \mathrm{C}$ ). Os tratamentos foram preparados em triplicatas. Alíquotas de $25 \mathrm{~mL}$ foram col etadas da solução dos tratamentos após 5, 10, 30, 120, 720 e $1.440 \mathrm{~min}$ de agitação em agitador horizontal. As soluções col etadas foram depositadas em tubos de cintilaçãolíquida ondese acrescentaram $0,5 \mathrm{~mL}$ de sol ução de $\mathrm{CaCl}_{2}$ 0,01 mol L-1 mais $7,5 \mathrm{~mL}$ de coquetel cintilante (Sigma). As amostras foram levadas ao contador decintilaçãolíquida (BECKMAN, model o LS6000I C) do Departamento deSol os da UFV para análise da radiatividade. $\mathrm{O}$ experimento foi realizado em temperatura ambiente $\left(18 \pm 2^{\circ} \mathrm{C}\right)$.
Os cálculos de adsorção foram feitos a partir da diferença dos val ores deradiotividademedidos em CPM (contagens por minuto) nas soluções sem solo (branco), antes de sua adição nas amostras e na solução de equilíbrio após os tempos de agitação. Estes val ores foram transformados em \% de atrazina adsorvida do total deatrazina adicionada inicialmente.

\section{RESULTADOS E DISCUSSÃO}

\section{Caracterização dos solos}

Os resultados da caracterização física e mineralógica dos sol os utilizados nos experimentos são apresentados no quadro 1; as características químicas, no quadro 2, eas características das frações orgânicas, no quadro 3. As duas amostras de Latossolo Roxo (manejo convencional e plantio dir eto) não diferiram quantoàs características físicas emineralógicas. Por serem solos de manejo diferenciado, algumas diferenças químicas foram observadas. A principal diferença foi a maior capacidade de troca de cátions (CTC) total e efetiva no solo sob plantio direto (Quadro 2), pois estesistema leva aoaumento do teor de carbono orgânico do solo (Sidiras et al., 1982) inclusive em Latossolo Roxo (Hernani et al., 1999), sendo a fração orgânica a grande responsável pela CTC. O nitrogênio total no solo sob plantio direto também foi maior.

O aumento da matéria orgânica em sol os beneficia a estruturação do solo, aumentando a porosidade e permeabilidade, pela presença demaior conexãoentre os macroporos. Com base na mi croestrutura descrita nas seções finas dos solos estudados (Figura 1), o manejo de plantio di reto resultou em maior soldagem

Quadro 1. Características físicas e mineralógicas das amostras de Latossolo Roxo utilizadas nos experimentos de mineralização e adsorção

\begin{tabular}{|c|c|c|c|c|c|}
\hline Profundidade & Areia & Silte & Argila & Classe textural & Mineralogia da argila(1) \\
\hline \multirow[t]{2}{*}{$\mathrm{cm}$} & 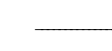 & ag kg-1 & - & & \\
\hline & \multicolumn{4}{|c|}{ Plantio convencional } & \\
\hline $0-10$ & 38 & 17 & 45 & Argila & $\mathrm{Gb}>\mathrm{Hm}>\mathrm{Gt}>\mathrm{Ct}$ \\
\hline $30-40$ & 30 & 10 & 60 & Muito argilosa & $\mathrm{Gb}>\mathrm{Hm}>\mathrm{Gt}>\mathrm{Ct}$ \\
\hline \multirow[t]{2}{*}{$70-80$} & 28 & 02 & 70 & Muito argilosa & $\mathrm{Gb}>\mathrm{Hm}>\mathrm{Gt}>\mathrm{Ct}$ \\
\hline & \multicolumn{4}{|c|}{ Plantio direto } & \\
\hline $0-10$ & 30 & 24 & 46 & Argila & $\mathrm{Gb}>\mathrm{Hm}>\mathrm{Gt}>\mathrm{Ct}$ \\
\hline $30-40$ & 27 & 12 & 61 & Muito argilosa & $\mathrm{Gb}>\mathrm{Hm}>\mathrm{Gt}>\mathrm{Ct}$ \\
\hline $70-80$ & 21 & 09 & 70 & Muito argilosa & $\mathrm{Gb}>\mathrm{Hm}>\mathrm{Gt}>\mathrm{Ct}$ \\
\hline
\end{tabular}

(1) Gb: gibbsita; Hm: hematita; Gt: goethita; Ct: caulinita. 
Quadro 2. Características químicas das amostras de Latossolo Roxo utilizadas nos experimentos de mineralização e adsorção

\begin{tabular}{|c|c|c|c|c|c|c|c|c|c|}
\hline Profundidade & $\mathbf{p H}$ & $\mathbf{P}$ & $\mathbf{K}$ & $\mathrm{Ca}$ & Mg & Al & $\mathbf{H}+\mathbf{A l}$ & CTC efet. $_{\text {. }}$ & CTC total. \\
\hline \multicolumn{10}{|c|}{$-\mathrm{mg} \mathrm{dm}^{-3}-$} \\
\hline \multicolumn{10}{|c|}{ Plantio convencional } \\
\hline $0-10$ & 6,9 & 73,0 & 134 & 5,7 & 0,7 & 0,0 & 0,6 & 6,7 & 7,3 \\
\hline $30-40$ & 6,3 & 3,0 & 134 & 3,0 & 0,6 & 0,0 & 2,4 & 3,9 & 6,3 \\
\hline $70-80$ & 6,2 & 1,5 & 86 & 2,9 & 0,3 & 0,0 & 2,1 & 3,4 & 5,5 \\
\hline \multicolumn{10}{|c|}{ Plantio direto } \\
\hline $0-10$ & 5,9 & 28,0 & 238 & 5,3 & 1,4 & 0,0 & 3,9 & 7,4 & 11,3 \\
\hline $30-40$ & 6,0 & 4,6 & 179 & 4,7 & 0,4 & 0,0 & 3,6 & 5,6 & 9,2 \\
\hline $70-80$ & 6,3 & 0,8 & 129 & 2,7 & 0,6 & 0,0 & 1,5 & 3,6 & 5,1 \\
\hline
\end{tabular}

Quadro 3. Características das frações orgânicas cabono e nitrogênio total das amostras de Latossolo Roxo estudadas nos experimentos de mineralização e adsorção

\begin{tabular}{|c|c|c|c|c|c|}
\hline Profundidade & Húmico & \multicolumn{2}{|c|}{ Fúlvico Humina } & C total & N-total \\
\hline $\mathrm{cm}$ & 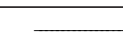 & 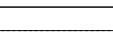 & $\operatorname{dag} \mathrm{kg}^{-1}$ & & \\
\hline \multicolumn{6}{|c|}{ Plantio convencional } \\
\hline $\begin{array}{r}0-10 \\
30-40\end{array}$ & $\begin{array}{l}0,19 \\
0,13 \\
0\end{array}$ & $\begin{array}{l}0,30 \\
0,26\end{array}$ & $\begin{array}{l}1,04 \\
0,47 \\
0\end{array}$ & $\begin{array}{l}1,83 \\
0,82 \\
0\end{array}$ & $\begin{array}{l}0,12 \\
0,06\end{array}$ \\
\hline \multicolumn{6}{|c|}{ Plantio direto } \\
\hline $\begin{array}{r}0-10 \\
30-40 \\
70-80\end{array}$ & $\begin{array}{l}0,56 \\
0,31 \\
0,067\end{array}$ & $\begin{array}{l}0,64 \\
0,44 \\
0,23\end{array}$ & $\begin{array}{l}1,12 \\
0,76 \\
0,47\end{array}$ & $\begin{array}{l}2,4 \\
1,48 \\
0,85\end{array}$ & $\begin{array}{l}0,21 \\
0,13 \\
0,07\end{array}$ \\
\hline
\end{tabular}

dos microagregados na superfície $(0-10 \mathrm{~cm})$ e mel hor estruturação em subsuperfície, em relação ao manejo convencional. Nas seções-finas, notou-sea coal escência deagregados entre 30 e 40 cm nomanejoconvencional, resultante do pé-de-grade, comum em L atossolo Roxo cultivado. Esta aparente inversão na formação de camadas adensadas pode provocar fluxos hídricos diferenciados entre os sistemas (Curmi et al., 1994), com efeito sobrea dinâmica do herbicida.

\section{Mineralização de atrazina no solo}

A mineral ização deatrazina foi maior no $L$ atossolo Roxo sob plantio direto (Figura 2 ) em relação ao Latossol o Roxosob plantio convencional (Figura 3) nas três profundidades estudadas. A curva de

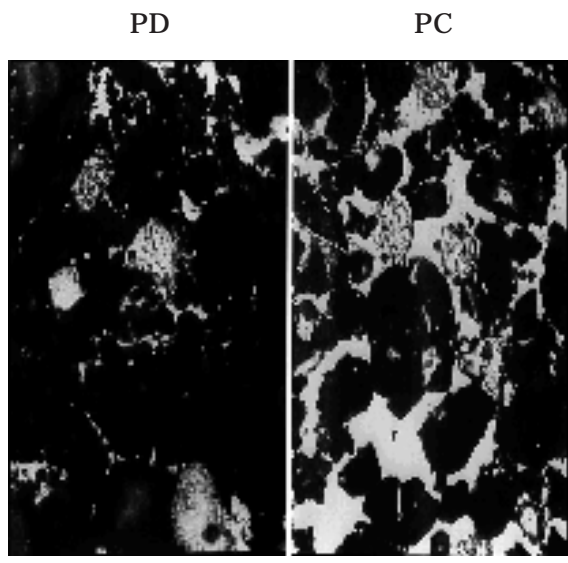

0-10
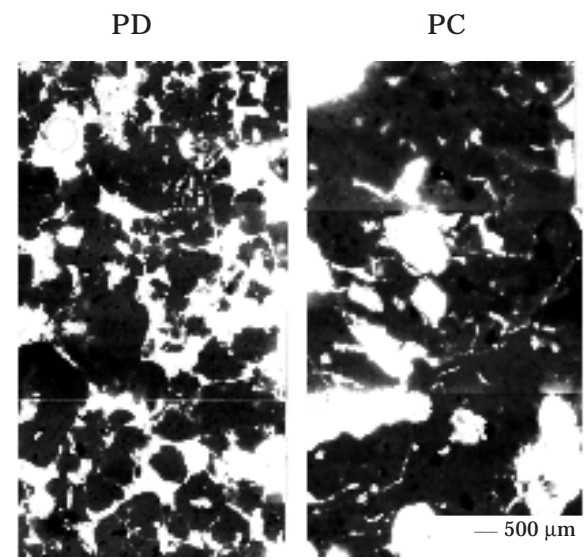

30-40

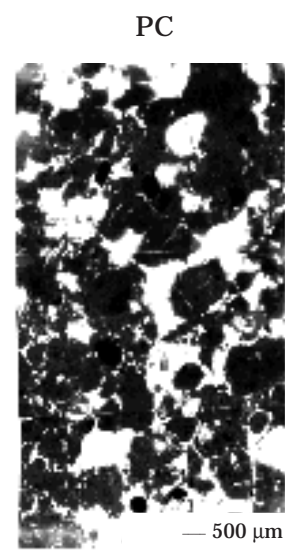

$70-80$

PROFUNDIDADE, $\mathrm{cm}$

Figura 1. Seções finas de um Latossolo Roxo em diferentes profundidades (plantio direto PD e plantio convencional PC). 
mineral ização de atrazina mostrou-se semel hanteà curva de crescimento de população microbiana. Observou-seuma fase inicial (faselag) deausência de mineral ização, fase que pode ser devida à adaptação microbiana para produção de enzimas responsáveis pela quebra da mol écula. Logo após, ocorreu o aumento da mineral ização deatrazina que refleteo orescimento exponencial da populaçãomi đorobiana (faseexponencial). Após a fase exponencial, observou-se um declínio da mineralização com tendência da curva de se tornar constante, ou seja, com mi neralização nula.

A adição de substratos orgâni cos após 100 dias de incubação (Figuras 2b e 3b) aumentou a taxa de mineral ização de atrazina, apresentando novamente uma fase exponencial com tendência no final do tempo de incubação a uma fase estacionária. É possível quea adição destes substratos tenha ativado a população microbiana por causa da introdução de nutrientes para os microrganismos, além de aumentar a mineralização de atrazina em maior profundidade em relação à superfície. I sso pode ser devido ao maior crescimento da população microbiana mais adaptada em mineralizar a atrazina sem que haja maior competição entre as espécies microbianas. Resultado semelhante foi encontrado por Goring \& Hamaker (1972), ao observarem que o enriquecimento do sol o com gl icose provocou uma aceleração na velocidade de degradação de atrazina.

Observando o comportamento de mineral ização em ambos os sol os, verificou-se que a faselag do LR sob plantio direto foi mais longa quea faselag do solo sob plantio convencional. Este fato evidencia uma maior adaptação dos mi crorganismos em mineralizar atrazina no sol o sob manejo convencional, talvez por ser a atrazina aplicada mais freqüentementeem sol os sob manejo convencional que em solos sob plantio direto. A atrazina, um herbicida pré-emergente, revel ou maior efi ciência quando aplicada diretamente sobre osol o mecanizado (Rodrigues \& Almeida, 1995).
Segundo Albuquerque(1995), a taxa demineralização de atrazina foi duas vezes maior, e sem presença da faselag, no solo onde este herbicida foi previamente aplicado, comparada à do mesmo sol o não cultivado por 15 anos, dada a necessidade de a população adaptar-se à atrazina, o que reforça a presente constatação.

O fato de a fase lag do plantio convencional ser menor que a do plantio direto pode estar associado à mai or disponi bilidade de carbono no plantio di reto, levando os microrganismos a decomporem atrazina somente após terem esgotado as fontes de carbono lábeis presentes.

Nas figuras 2 e 3, observa-sequea taxa demineralização de atrazina decaiu com a profundidade do solo. A mineralização correlacionou-se significativamente com o teor de matéria orgânica do solo, com o teor de nitrogênio e com as frações ácido húmico, fúlvico e humina (Quadro 4). A diferença entre as profundidades e entre os solos (plantio direto e convencional) deveu-se ao teor de matéria orgânica. O plantio direto, por ter maior teor de carbono orgânico tanto na superfície quanto na subsuperfície (Quadro 3), conferiu maior atividade microbiana e, conseqüentemente, maior mineralização de atrazina. Hirata \& Ruegg (1985), estudando a mineralização do carbaril em diferentes solos, encontraram maior taxa de mineralização nos solos com maior teor de matéria orgânica. Esses autores atribuíram esta maior taxa demineralização aofato de osol ocom maior teor de matéria orgânica permitir maior população microbiana.

Nos resultados de mineral ização de atrazina em ambos os sistemas de manejo, verificou-seuma baixa taxa demineralização deatrazina em relação ao total deatrazina adicionada deacordo com a profundidade. Resultados semel hantes foram obtidos por J ohnson \& Fuhrmann (1993) que, estudando atrazina e metolachlor em profundidade, concluíram ser o potencial de mineralização de atrazina em

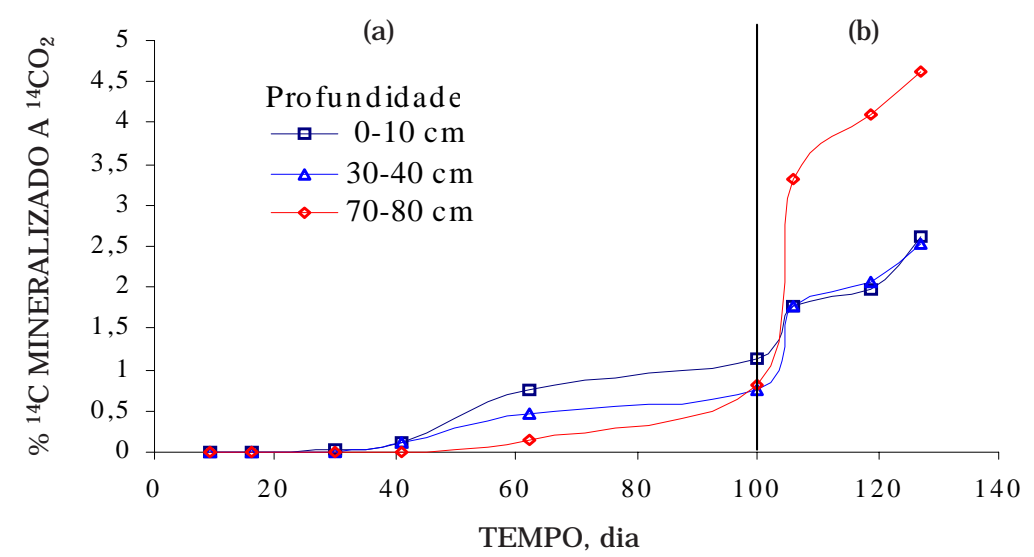

Figura 2. Mineralização de atrazina em amostras de Latossolo R oxo sob sistema plantio direto. (a) período de incubação sem adi ção de ami do e (b) período com adi ção de ami do. 


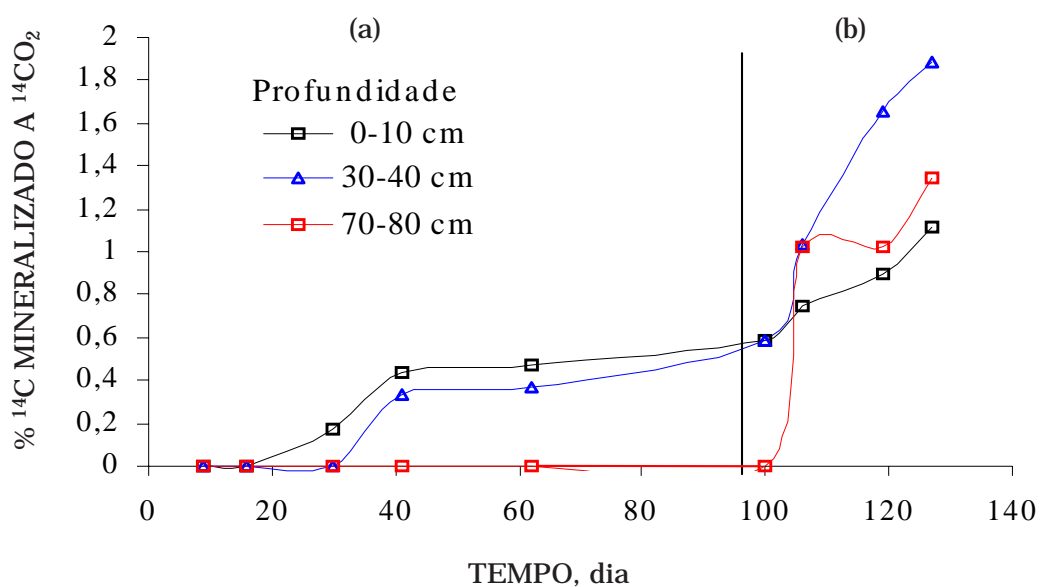

Figura 3. Mineralização de atrazina em amostras de Latossolo Roxo sob sistema de cultivo convencional. (a) período de incubação sem adição de composto orgânico e (b) período com adi ção de composto orgânico.

Quadro 4. Correlação entre os componentes orgânicos do LatossoloRoxocoma mineralização de atrazina $-{ }^{14} \mathrm{C}$ medida aos 62 dias de incubação

\begin{tabular}{llc}
\hline Componente do solo & Equação ajustada & r \\
\hline Carbono total & $\hat{Y}=-0,1081+0,3557^{* *} x$ & 0,91 \\
Nitrogênio total & $\hat{Y}=-0,0597+4,0129^{* *} x$ & 0,91 \\
Fração ácido húmico & $\hat{Y}=-0,0942+1,2643^{* *} x$ & 0,92 \\
Fração ácido fúlvico & $\hat{Y}=-0,1176+1,4153^{*} x$ & 0,89 \\
Fração humina & $\hat{Y}=-0,1522+0,7382^{*} x$ & 0,89
\end{tabular}

**, * Significativos a 1 e $5 \%$, respectivamente.

profundidademuito baixa, principalmentenosubsolo. Albuquerque (1995) atribuiu essa baixa ou nula mineralização em profundidade à ausência ou ao pequenonúmerode microrganismos responsáveis pela mineralização deatrazina nesse ambiente.

Acredita-sequea baixa mineral ização deatrazina esteja associada à grande estabilidade química e biológica do herbicida, conforme demonstram inúmeros trabalhos em países de clima temperado (Koskinen \& Clay, 1997; Clark et al., 1999). A atividademi crobiana mais el evada em áreas tropicais talvez nãoseja tão efetiva no caso da atrazina, trazendo preocupação com o comportamento e o destino do herbicida em condições brasileiras.

\section{Cinética de adsorção de atrazina no solo}

As figuras 4, 5 e 6 mostram a adsorção deatrazina ao longo do tempo em materiais de $L R$. As curvas $A$ eB da figura 4 mostram a adsorção de atrazina para os tratamentos com matéria orgânica. Nessas curvas, verifica-se uma rápida adsorção inicial, aproximadamente $35 \%$ do total adicionado após $2 \mathrm{~h}$, ocorrendo baixo incrementona adsorção, atingindo um platôlogo após essetempo.

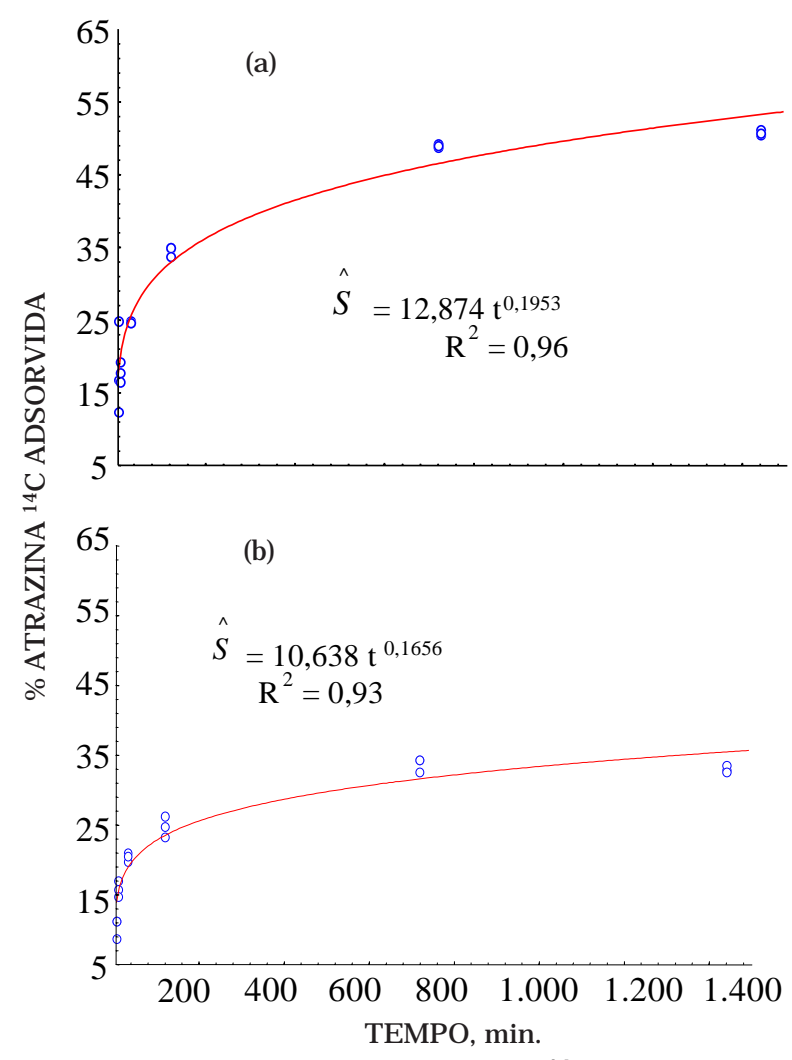

Figura 4. Percentagem de atrazina $-{ }^{14} \mathrm{C}$ adsorvida do total adicionado em amostras de LR. Solo (a) e argila com matéria orgânica (b). 


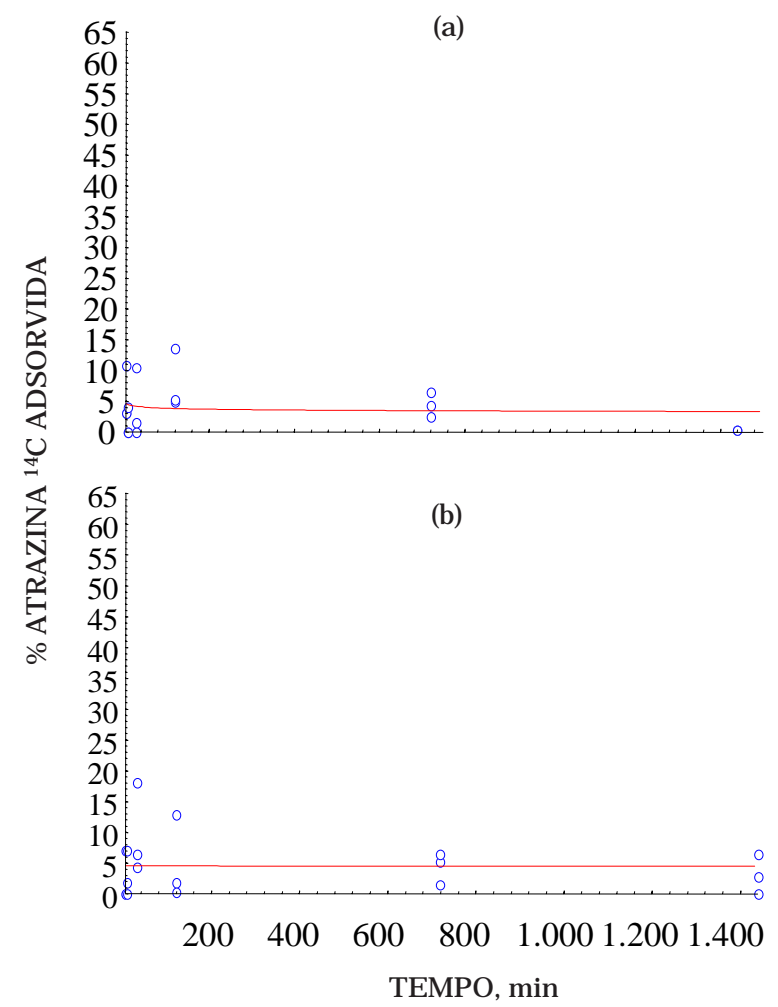

Figura 5. Percentagem de atrazina- ${ }^{14} \mathrm{C}$ adsorvida do total adicionado em materiais de Latossolo Roxo. Solo com matéria orgânica removida (a) e argila com matéria orgânica removida (b).

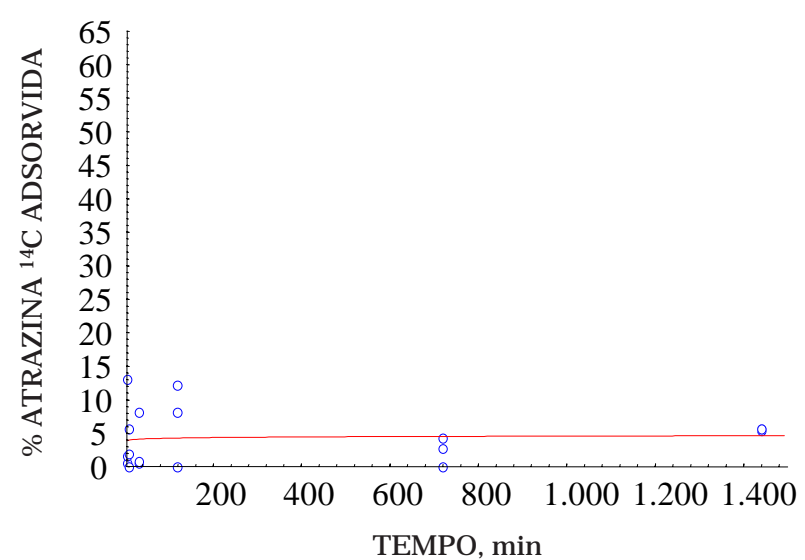

Figura 6. Percentagem de atrazina- ${ }^{14} \mathrm{C}$ adsorvida do total adicionado em materiais de Latossolo Roxo com matéria orgânica e óxidos de ferro removidos.

Green \& Karickhoff (1990) concluíram que o processo de adsorção ao longo do tempo apresenta um componente rápido e outro mais lento, conforme o movimento do soluto para pontos deadsorção menos acessíveis dos colóides do solo. K han (1973), estudando a cinética de adsorção do herbicida 2,4-D pel a fração ácido húmico, encontrou resultados semel hantes. Esse autor atribuiu a adsorção inicial à interação das moléculas do herbicida sobre a superfície do colóide. A pós um período de tempo, o processo tornou-se mais lento, graças à difusão do composto para o interior das partículas da fração ácido húmico.

Os resultados dos tratamentos sem matéria orgânica podem ser observados nas figuras 5 e 6 . Uma pequena adsorção inicial (5\%) foi observada, não tendo sido verificado incremento de adsorção aolongo do tempo. Estefato indica que, nos materiais sem matéria orgânica, as possíveis ligações entre a atrazina e a fração mineral são instantâneas e de superfície, provavel menteatribuídas a forças de Van der Waals e pontes de hidrogênio, conforme relatos de Laird et al. (1994) e Celis et al. (1997). Percebeuse baixo teor de atrazina adsorvida quando a adsorção foi realizada em meio aquoso, onde as moléculas de água competiam com os herbicidas pel os mesmos locais de adsorção (Deuber, 1992; Laird et al., 1994).

No quadro 5, pel o contraste 1, verificou-se efeito significativo da matéria orgânica na adsorção de atrazina; pelo contraste 2 , não foi verificada diferença significativa de adsorção entre os tratamentos que continham óxidos de ferro e o desferrificado, demonstrando que, nas condições em que foram realizados os experimentos, os óxidos de ferro e alumínio, juntamente com a caulinita, não tiveram capacidade de adsorver atrazina. Pelo contraste 3 , confrontando os tratamentos argila sem matéria orgânica esolo sem matéria orgânica, verificou-seque não houve diferença significativa entre estes tratamentos. O sol o integral continha $45 \%$ de argila (Quadro 1), sendo as demais frações grosseiras (siltee areia) demenor superfícieespecífica. Percebeu-seque

Quadro 5. Valores dos contrastes das médias, em percentagem adsorvida do total adicionado de atrazi na- ${ }^{14} \mathrm{C}$, dos tempos de adsorção correspondente a 12 e 24 horas

\begin{tabular}{lc}
\hline \multicolumn{1}{c}{ Contraste } & Adsorção relativa \\
\hline & $\%$ \\
$\mathrm{C}_{1}:(\mathrm{sol}+\mathrm{argc} / \mathrm{mo})-(\mathrm{sol} / \mathrm{mo}+\mathrm{args} / \mathrm{mo})$ & $38,8^{* *}$ \\
$\mathrm{C}_{2}:(\mathrm{args} / \mathrm{Fe})$ - (args/mo) & $0,62^{\mathrm{ns}}$ \\
$\mathrm{C}_{3}:(\mathrm{args} / \mathrm{mo})-(\mathrm{sols} / \mathrm{mo})$ & $0,26^{\mathrm{ns}}$ \\
$\mathrm{C}_{4}:(\mathrm{sol})$ - (sols/ag) & $0,38^{\mathrm{ns}}$ \\
$\mathrm{C}_{5}:(\mathrm{sol})$ - (argc/mo) & $14,95^{* *}$ \\
C.V. (\%) & 9,7
\end{tabular}

** $\mathrm{e}^{\mathrm{ns}}$ : Significativo a $1 \%$ e não-significativo.

sol =solo integral; sols $/ \mathrm{mo}=$ sol o sem matéria orgânica; args $/ \mathrm{mo}$ = argila sem matéria orgânica; argc/mo = argila com matéria orgânica; $\operatorname{args} / \mathrm{Fe}=$ argila sem óxidos de ferro; sols/ag = solo integral sem agitação. 
as frações siltee areia do solo não adsorviam atrazina, bem como a baixa correl ação de adsorção de atrazina com a superfície específica da fração mineral desse solo. As implicações da contaminação de atrazina nas águas subsuperficiais em condições brasileiras merecem atenção pel o possível efeito carcinogênico, reportado recentemente(Birardar \& Rayburn, 1995).

O tratamento argila com matéria orgânica adsorveu significativamentemenos atrazina (35\%)que o solo integral (50\%) (contraste 5). Estefato foi devido à perda de material orgânico, principal-menteácido húmico efúlvico, no processoalcalino deseparação da fração argila. As ligações envolvidas entrea atrazina e a matéria orgânica devem-se tanto a pontes de hidrogênio(Hayes, 1970) quantoa ligações hidrofóbicas (Hance, 1969; Wang et al., 1991, Martin-Neto et al., 1994; Sposito et al., 1996).

\section{CONCLUSÕES}

1. A taxa de mineralização de atrazina a $\mathrm{CO}_{2}$ foi muito baixa, apresentando correl ações significativas com os teores de carbono, nitrogênio total e frações húmicas.

2. A mineralização de atrazina diminuiu em profundidade no Latossolo Roxo. O plantio direto mostrou mai or mineral ização de atrazina em virtude dos maiores teores de carbono. E m contrapartida, a fase lag do plantio convencional foi mais curta, evidenciando mai or adaptação mi crobiana nos solos onde oherbicida foi mais intensamente utilizado.

3. A adição de substratos orgânicos aumentou o processo de mineralização de atrazina no solo.

4. A atrazina contém um componente rápido de adsorção, em torno de $2 \mathrm{~h}$, e um componente mais lento que se estabelece até 12 h, no solo.

5. A matéria orgânica foi a principal responsável pela adsorção da atrazina aolongo do tempo, enquanto os óxidos de ferro e minerais de argila 1:1 não contribuíram para a adsorção.

6. Sol os agrícolas oxídicos, com baixoteor dematéria orgânica, não adsorvem atrazina em quantidades suficientes para evitar a contami nação subsuperficial, ondea molécula não pode ser mineralizada, trazendo riscos à saúde pública pela contaminação do lençol freático.

\section{LITERATURA CITADA}

AISLABIE, J . \& LLOYD-J ONES, G. A review of bacterial degration of pesticides. Aust. J. Soil Res., 33:925-42, 1995.
ALBUQUERQUE, M.A. Degradation of atrazine in soil and subsurface. Reading, University of Reading, 1995, 164p. (Tese de Doutorado)

ANDERSON, J.U. An improved pretreatment for mineralogical analysis of samples containing organic matter. Clays Clays Miner., 10:330-388, 1963.

ARMSTRONG, D.E.; CHESTERS, G. \& HARRIS, R.F. Atrazine hydrolysis in soil. Soil Sci. Soc Am. Proc., 31:61-66, 1967.

BAILEY, G.W. \& WHITE, J.L. Factors influencing the adsorption, dessorption and movement of pesticide in soil. Res. Rev., 32:29-92, 1970.

BIRARDAR, D.P. \& RAYBURN, A.L. Chromosomal damage induced by herbicide contamination at concentrations observed in public water supplies. J. Environ. Qual., 24:1222-1225, 1995.

BOLLAG, J .M. \& LIU, S.Y. Biological transformation processes of herbicides. In: CHENG, H.H., ed. Pesticides in the soil environment: processes, impacts and modelling. Madison, Soil Science Society of America, 1990, p.169-203.

CELIS, R.; CORNEJ O, J .; HERMOSÍN, M.C. \& KOSKINEN, W.C. Sorption-desorption of atrazine and simazine by soil model colloidal components. Soil Sci. Soc. Am. J ., 61:436443, 1997.

CLARK, G.M.; GOOLSBY, D.A. \& BATTAGLIN, W.A. Seasonal and annual load of herbicides from the Mississipi River to the Gulf of Mexico. Environ. Sci. Technol., 33:981-986, 1999.

CURMI, P.; KERTZMAN, F.F. \& QUEIROZ NETO, J.P. Degradation of structure and hydraulic properties in an Oxisol under cultivation (Brazil). In: RINGROASEVOASE, A.J. \& HUMPHRHEYS, G.S., eds. Soil micromorphology: studies in management and genesis. Amsterdam, Elsevier:, 1994. p.569-581.

DEUBER, R. Métodos de controle de plantas daninhas. In: Ciências das plantas daninhas. J aboticabal, Universidade Estadual Paulista, 1992. p.48-109.

EMPRESA BRASILEIRA DE PESQUISA AGROPECUÁRIA EMBRAPA. Centro Nacional de Pesquisa de Solos. Manual de métodos de análises de solos. Rio de J aneiro, 1997. 212p.

FITZPATRICK, E.A.E.W. Soil microscopy and micromorphology. Chichester, J ohn Wiley, 1993. 304p.

GORING, C.A.I. \& HAMAKER, J.W. Organic chemicals in the environment. New York, M. Dekker, 1972. 440p.

GREEN, R.E. \& KARICKHOFF, S.W. Sorption estimates for modelling. In: CHENG, H.H., ed. Pesticides in the soil environment. Madison, Soil Science Society of America, 1990. p.169-203.

HANCE. R.J. Influence of $\mathrm{pH}$, exchangeable cation and presence of organic matter on the adsorption of some herbicides by montmorillonite. J. Soil Sci., 49:357-364, 1969.

HAYES, M.H.B. Adsorption of atrazine herbicides on soil organic matter, including a short review on soil organic matter chemistry. Residue Rev., 31:131-174, 1970. 
HAYES, M.H.B.; MACCARTHY, P. \& MALCOLM, R.L. The search for structure: setting the scene. In: HAYES, M.H.B., ed. Humic susbstances II: In search of structure. Chichester, J ohn Wiley, 1989. 764p.

HERNANI, L.C.; KURIHARA, C.H. \& SILVA, W.M. Sistemas de manejo de solo e perdas de nutrientes e matéria orgânica por erosão. R. Bras. Ci. Solo, 23:145-154, 1999.

HIRATA, R. \& RUEGG, E.F. Degradação do carbariL- ${ }^{14} \mathrm{C}$ em sol os modificados por oxidação da matéria orgânica eadição de glicose. Pesq. Agropec. Bras., 20:621-629, 1995.

J OHNSON, R.M. \& FUHRMANN, J J . Degradation of atrazine and metilachror in subsoils from an Atlantic Coastal Plain Watershed. Madison, Soil Science Society of America, 1993. p.27-31. (Special Publication, 32)

KHAN, S.U. Equilibrium and kinetic studies of the adsorption of 2,4-D and picloran on humic acid. Can. J. Soil Sci., 55:429-434, 1973.

KOSBINEN, W.C. \& CLAY, S.A. Factors affecting atrazine fate in north central U.S. Rev. Environ. Contam. Toxicol., 151:117-165. 1997.

LAIRD, D.A.; YEN, P.Y.; KOSKINEN, W.C.; STEINHEIMER, T.R. \& DOWDY, R.H. Sorption of atrazine on soil clay components. Environ. Sci. Technol., 28:1054-1061, 1994.

MARTIN-NETO, L.; VIEIRA, E.N. \& SPOSITO, G.L. Mechanism of atrazine sorption by humic acid: $A$ spectroscopic study. Envirom. Sci., 28:1867-1873, 1994.
MEHRA, O.P. \& J ACKSON, M.L. I ron oxide removal from soil and clays by a dothionite-citrate system buffered with sodium bicarbonate. Clays Clays Miner., 7:317-327, 1960.

RITTER, W.F.; SCARBOROUGH, R.W. \& CHIRNSIDE, E.A.E.M. Contamination of groundwater by triazine, metolaclhor and alachlor. J. Contam. Hidrol., 15:73-92, 1994.

RODRIGUES, B.N. \& ALMEIDA, F.S. Guia de herbicidas. 3.ed. Londrina, edição dos autores. 1995, 675p.

SIDIRAS, N.; HENKLAIN, J .C. \& DERPSCH, R. Comparation of three different tillage systems with respect to aggregate stability, the soil and water conservation and the yields of soybean and wheat on an Oxisol. In: INTERNATIONAL SOIL TILLAGE RESEARCH ORGANIZATION CONFERENCE 9., Ojisek, 1982. Anais. Ojisek, ISTRO, 1982. p.537-544.

SPOSITO, G.L. \& MARTIN NETO, A.Y. Atrazine complexation by soil humic acids. J . Environ. Qual., 25:1203-1209, 1996.

WANG, Z.-D.; GAMBLE, D.S. \& LANGFORD, C.H. Interaction of atrazine with Laurentian humic acid. Anal. Chim. Acta., 244:135-143, 1991.

WORTHING, C.R. \& HANCE, R.J. The pesticide manual. Surrey, Br. Crop Protection Council, UK. 1991. 350p.

YOEMANS, J.C. \& BREMNER, J.M. A rapid and precise method for routine determination of organic carbon in soil. Comm. Soil Sci. Plant Anal., 19:1467-1476, 1988. 